\title{
Proprotein convertase subtilisin/kexin type 9 enzyme inhibitors: An emerging new therapeutic option for the treatment of dyslipidemia
}

\author{
Faizan Mazhar ${ }^{1}$, Nafis Haider ${ }^{1,2}$ \\ ${ }^{1}$ Department of Basic Medical Sciences, Prince Sultan Military College of Health Sciences, King Fahd Military Medical Complex, Dhahran, \\ Saudi Arabia, ${ }^{2}$ Department of Pharmaceutical Biotechnology, Faculty of Pharmacy, Jamia Hamdard, New Delhi, India
}

\begin{abstract}
Received: 29-04-2016
Revised: 17-07-2016

Accepted: 26-10-2016

ABSTRACT

The treatment of hypercholesterolemia entered in a new phase of development with the introduction of proprotein convertase subtilisin/kexin type 9 (PCSK9) inhibitors in the market. The Food and Drug Administration and European Medicines Agency recently approved the alirocumab and evolocumab, subcutaneously injectable monoclonal antibody every 2 or 4 weeks against PCSK9, for the treatment of hypercholesterolemia in patients with intolerance or inadequate response to statins, especially for the secondary prevention or in the case of familial hypercholesterolemia. This decision is based on several clinical trials demonstrating that inhibitors of PCSK9 lower the low-density lipoprotein cholesterol compared to placebo while studies are underway to assess their role in secondary prevention of major cardiovascular events.
\end{abstract}

Key words: Alirocumab, dyslipidemia, evolocumab, proprotein convertase subtilisin/kexin type 9

\section{INTRODUCTION}

Cardiovascular (CV) disease remains the leading cause of death and one of the main causes of morbidity in our era. ${ }^{[1]}$ Elevated levels of total cholesterol and specifically cholesterol linked to low-density lipoprotein (LDL) have a positive and growing association for the development of ischemic heart disease and CV mortality. ${ }^{[2]}$ Lipid theory of atherosclerosis has had an endorsement from a large number of powerful clinical trials,

\begin{tabular}{|l|l|}
\hline \multicolumn{2}{|c|}{ Access this article online } \\
\hline Quick Response Code: & Website: \\
\hline & www.jpharmacol.com \\
\cline { 2 - 3 } & \\
\hline
\end{tabular}

Address for correspondence:

Nafis Haider, Department of Pharmaceutical Biotechnology,

Faculty of Pharmacy, Jamia Hamdard, New Delhi, India.

E-mail: nafispharma@gmail.com which has been consistently shown that a reduction of total cholesterol (LDL-cholesterol [LDL-C]) is associated with a reduction of the risk of coronary heart disease (CHD), stroke, and CV mortality. ${ }^{[3]}$ Undoubtedly, most of the categorical data for the primary and secondary prevention of $\mathrm{CV}$ disease obtained in clinical trials are related to statins. In addition, intensive treatment with statins has shown superior protective effects compared to moderate or low statin dosing. ${ }^{[4]}$ Despite the unquestionable success of statins, most trials show a relative risk reduction of around $30 \%$. The other $70 \%$ which is usually referred as "residual risk," ${ }^{[5,6]}$ perhaps it would be more appropriate to call it "persistent risk."

This is an open access article distributed under the terms of the Creative Commons Attribution-NonCommercial-ShareAlike 3.0 License, which allows others to remix, tweak, and build upon the work non-commercially, as long as the author is credited and the new creations are licensed under the identical terms.

For reprints contact: reprints@medknow.com 


\section{Mazhar and Haider: Role of PCSK9 inhibitors in dyslipidemia treatment}

For a multifactorial disease such as arteriosclerosis, it is unrealistic to control a disease completely by targeting a single risk factor; therefore, it is still reasonable to suggest lipid-lowering therapy that offers potentially protective options. ${ }^{[7]}$ In this regard, it is worth to mention that epidemiological studies shown that raising high-density lipoprotein (HDL) cholesterol shows a clear association in reducing CV complications. ${ }^{[2]}$ Unfortunately, most of the therapeutic trials have resulted in disappointing results that were aimed at the elevation of HDL, particularly recent trials in patients already taking statins and using a various pharmacological class such as fibrates, niacin, or inhibitors of cholesteryl ester transfer protein. ${ }^{[8]}$

Recent genetic studies have questioned the biological role of lowering HDL cholesterol in the development of arteriosclerosis. ${ }^{[9]}$ Mendelian randomization studies offer an opportunity to evaluate the relevance of different mutations that modify different risk factors and their impact on the development of atherosclerosis from birth through natural experiments. ${ }^{[10]}$ For example, these studies support the importance of lipoprotein(a) but not of C-reactive protein in the etiology of arteriosclerosis. Similar studies have shown that genetic alterations from birth that modify the levels of HDL cholesterol are not associated with an increase or decrease in CV complications. ${ }^{[9,10]}$ These studies do not deny that HDL cholesterol levels are markers of vascular risk but questioned the relevance of the same in the etiology of the vascular lesion. In contrast, Mendelian randomization studies do show a clear and consistent relationship between various genes that modify LDL-C levels and the development of arteriosclerosis, ${ }^{[9]}$ and very remarkable genes involved in the synthesis (HMG-CoA reductase) and absorption Niemann-Pick C1-like 1 cholesterol, and degradation of LDL receptor (proprotein convertase subtilisin/kexin type 9 [PCSK9]). ${ }^{[1,12]}$

Recent European and American guidelines on dyslipidemia differ in several respects, but they agree that LDL-C as a therapeutic target and statins as a first-line treatment. ${ }^{[13,14]}$ Therefore, the most pressing question arises whether we can achieve greater reductions in LDL-C with other treatment options and whether these will result in a reduction of $\mathrm{CV}$ complications. The benefits and limitations of statin therapy have been extensively evaluated. The unquestionable success of statins has triggered the paradoxical situation that almost negate the potential therapeutic value of other lipid-lowering drugs. ${ }^{[15]}$ However, one aspect that has received little attention is that statins trigger counter-regulation of genes that attenuate its lipid-lowering effect. Among them, PCSK9 and NP1CL1 are genes that regulate proteins mentioned above, and that, in turn, are epidemiologically associated with changes in LDL-C levels and CV morbidity. ${ }^{[10-12]}$

\section{MECHANISM OF ACTION}

This class of drugs inhibits the PCSK9 enzyme, which plays a major role in the breakdown of hepatic LDL receptors and is typically upregulated in the presence of statins. The inhibition of this enzyme enables more efficient hepatic uptake of LDL, decreasing serum LDL levels by $>50 \%$ in most cases. This mechanism was discovered barely more than a decade ago, and already targeting this pathway are five agents: Alirocumab, evolocumab, and three additional agents in the pipeline.

\section{ODYSSEY LONG TERM TRIAL}

One recent major trial, ODYSSEY LONG TERM, supports the addition of alirocumab to statin therapy to reduce both lipid parameters and possibly, overall CV events. More than 2300 patients with high atherosclerotic CV disease (ASCVD) risk who were receiving maximally tolerated statin therapy were assigned to either alirocumab or placebo as adjunct therapy. ${ }^{[16,17]}$ At the end of the 78-week study, patients who had received alirocumab experienced a statistically significant $62 \%$ LDL decrease versus the placebo group; a post hoc analysis noted a statistically significant lower rate of major adverse CV events in the alirocumab group versus placebo. ODYSSEY ALTERNATIVE trial showed that alirocumab produced greater LDL-C reductions than ezetimibe in statin-intolerant patients, with fewer skeletal muscle adverse events versus atorvastatin. ${ }^{[18]}$

Several Phase III clinical trials explored the use of evolocumab as part of various therapies with a range of possible dosing strategies. The MENDEL-2 trial focused on about 600 patients with $\mathrm{LDL} \leq 190 \mathrm{mg} / \mathrm{dL}$ and 10 -year CHD risk (per the Framingham risk score) of $10 \%$ or less in a six-arm design, comparing evolocumab injections both biweekly and monthly with placebo and ezetimibe. ${ }^{[19]}$ Overall, both doses of evolocumab resulted in an LDL decrease of about 56\% versus placebo and an LDL decrease of about 39\% versus ezetimibe, with no difference between the groups in terms of adverse events.

\section{DESCARTES TRIAL}

The DESCARTES trial investigated 900 patients who were receiving diet alone, atorvastatin $10 \mathrm{mg}$ daily, or atorvastatin $80 \mathrm{mg}$ daily with or without ezetimibe and randomized those patients to either monthly evolocumab or placebo. ${ }^{[20]}$ The results demonstrated statistically significant decreases in LDL with evolocumab versus placebo in all groups, with an overall reduction of $57 \%$ from baseline in the entire cohort, which persisted even in the presence of high-intensity statin therapy. Two extension studies conducted with patients 


\section{Mazhar and Haider: Role of PCSK9 inhibitors in dyslipidemia treatment}

who had participated in previous Phase II or III trials of evolocumab-OSLER-1 and OSLER-2 - compared the addition of evolocumab with standard therapy for hyperlipidemia versus the use of standard therapy alone. ${ }^{[21]}$ The investigators defined standard therapy as an appropriate guideline-directed therapy for the level of ASCVD risk present in each patient; almost $70 \%$ of the patients in the study were receiving statin therapy. Evolocumab decreased LDL by $61 \%$ in the overall population and statistically significantly reduced the incidence of CV events at 1 year by $53 \%$ versus standard therapy alone. Finally, evolocumab has been studied in both statin-intolerant patients and heterozygous familial hypercholesterolemia (HeFH) with a $>50 \%$ LDL reduction in those patient populations as well. ${ }^{[22,23]}$

\section{PRESENT STATUS}

In mid-2015, both alirocumab and evolocumab received Food and Drug Administration (FDA) approval for use in patients with $\mathrm{HeFH}$ and patients with clinical ASCVD who are receiving maximally tolerated statin therapy and who still require further LDL-lowering. The recommended starting dose for alirocumab is $75 \mathrm{mg}$ administered subcutaneously once every 2 weeks, since the majority of patients achieve sufficient LDL-C reduction with this dosage. If the LDL-C response is inadequate, the dosage may be increased to the maximum dosage of $150 \mathrm{mg}$ administered every 2 weeks. Measure LDL-C levels within 4-8 weeks of initiating or titrating alirocumab to assess response and adjust the dose, if needed. Evolocumab also received approval for use in patients with homozygous familial hypercholesterolemia. Evolocumab dose for primary hyperlipidemia with established clinical ASCVD or HeFH is $140 \mathrm{mg}$ every 2 weeks or $420 \mathrm{mg}$ once monthly in abdomen, thigh, or upper arm. Because large-scale, long-term clinical outcomes data are not expected until 2017 , the benefit-risk ratio has not yet been fully elucidated, and the role for addressing residual ASCVD risk remains unclear. However, for practitioners with limited options, this class of agents represents a novel approach to managing difficult dyslipidemias.

Part 2 of the National Lipid Association (NLA) recommendations, the only guideline to address this class thus far, suggests that these agents could be used in patients with ASCVD and LDL of $100 \mathrm{mg} / \mathrm{dL}$ or greater who are receiving maximally tolerated statin with or without ezetimibe therapy, as well as in patients with $\mathrm{HeFH}$ without ASCVD but with LDL of $130 \mathrm{mg} / \mathrm{dL}$ or greater who are on maximally tolerated (high-intensity) statins. The NLA also notes that those agents could be considered in patients who are statin intolerant - although not FDA approved for this indication. Both agents are administered as subcutaneous injections and are well tolerated overall, with the most predominant adverse effects being mild injection site reactions and muscle aches.
Neurocognitive events were reported more frequently with both drugs than with placebo. This adverse effect will be the subject of observation in ongoing studies. We still await the results of 4 ongoing large placebo-controlled Phase III trials investigating whether PCSK9 inhibitors on background of statin therapy reduce $\mathrm{CV}$ events.

\section{CONCLUSION}

The availability of new high potency lipid-lowering agents, particularly anti-PCSK9 monoclonal antibody opens a new horizon in the prevention and treatment of atherosclerosis. These are the valuable option particularly in those patients who are intolerant to statin or inadequately controlled lipid targets despite intensive statin treatment. Long-term studies are underway to assess the impact on $\mathrm{CV}$ events.

\section{Financial support and sponsorship \\ Nil.}

\section{Conflicts of interest}

There are no conflicts of interest.

\section{REFERENCES}

1. Chauhan S, Aeri BT. Prevalence of cardiovascular disease in India and it is economic impact - A review. Int J Sci Res Publ 2013;3:1-5.

2. Prospective Studies Collaboration, Lewington S, Whitlock G, Clarke R, Sherliker P, Emberson J, et al. Blood cholesterol and vascular mortality by age, sex, and blood pressure: A meta-analysis of individual data from 61 prospective studies with 55,000 vascular deaths. Lancet 2007;370:1829-39.

3. Unit ES. Efficacy and safety of cholesterol-lowering treatment: Prospective meta-analysis of data from 90056 participants in 14 randomised trials of statins. Lancet 2005;366:1267-78.

4. Cholesterol Treatment Trialists' (CTT) Collaboration, Baigent C, Blackwell L, Emberson J, Holland LE, Reith C, et al. Efficacy and safety of more intensive lowering of LDL cholesterol: A meta-analysis of data from 170,000 participants in 26 randomised trials. Lancet 2010;376:1670-81.

5. Joshi PH, Martin SS, Blumenthal RS. The remnants of residual risk. J Am Coll Cardiol 2015;65:2276-8.

6. Sampson UK, Fazio S, Linton MF. Residual cardiovascular risk despite optimal LDL cholesterol reduction with statins: The evidence, etiology, and therapeutic challenges. Curr Atheroscler Rep 2012;14:1-10.

7. Brown MS, Goldstein JL. Heart attacks: Gone with the century? Science 1996;272:629.

8. Keene D, Price C, Shun-Shin MJ, Francis DP. Effect on cardiovascular risk of high density lipoprotein targeted drug treatments niacin, fibrates, and CETP inhibitors: Meta-analysis of randomised controlled trials including 117,411 patients. BMJ 2014;349:g4379.

9. Voight BF, Peloso GM, Orho-Melander M, Frikke-Schmidt R, Barbalic M, Jensen MK, et al. Plasma HDL cholesterol and risk of myocardial infarction: A mendelian randomisation study. Lancet 2012;380:572-80.

10. Nordestgaard BG, Tybjærg-Hansen A. Genetic determinants of LDL, lipoprotein(a), triglyceride-rich lipoproteins and HDL: Concordance and discordance with cardiovascular disease risk. Curr Opin Lipidol 2011;22:113-22.

11. Myocardial Infarction Genetics Consortium Investigators, Stitziel NO, Won HH, Morrison AC, Peloso GM, Do R, et al. Inactivating mutations in NPC1L1 and protection from coronary heart disease. N Engl J Med 2014;371:2072-82.

12. Ference BA, Majeed F, Penumetcha R, Flack JM, Brook RD. Effect of 


\section{Mazhar and Haider: Role of PCSK9 inhibitors in dyslipidemia treatment}

naturally random allocation to lower low-density lipoprotein cholesterol on the risk of coronary heart disease mediated by polymorphisms in NPC1L1, HMGCR, or both: A $2 \times 2$ factorial Mendelian randomization study. J Am Coll Cardiol 2015;65:1552-61.

13. Perk J, De Backer G, Gohlke H, Graham I, Reiner Z, Verschuren M, et al. European guidelines on cardiovascular disease prevention in clinical practice (version 2012). The Fifth Joint Task Force of the European Society of Cardiology and Other Societies on Cardiovascular Disease Prevention in Clinical Practice (constituted by representatives of nine societies and by invited experts). Eur Heart J 2012;33:1635-701.

14. Stone NJ, Robinson JG, Lichtenstein AH, Goff DC Jr., Lloyd-Jones DM, Smith SC Jr., et al. Treatment of blood cholesterol to reduce atherosclerotic cardiovascular disease risk in adults: Synopsis of the 2013 American College of Cardiology/American Heart Association cholesterol guideline. Ann Intern Med 2014;160:339-43.

15. Dubuc G, Chamberland A, Wassef H, Davignon J, Seidah NG, Bernier L, et al. Statins upregulate PCSK9, the gene encoding the proprotein convertase neural apoptosis-regulated convertase-1 implicated in familial hypercholesterolemia. Arterioscler Thromb Vasc Biol 2004;24:1454-9.

16. Robinson JG, Farnier M, Krempf M, Bergeron J, Luc G, Averna M, et al. Efficacy and safety of alirocumab in reducing lipids and cardiovascular events. N Engl J Med 2015;372:1489-99.

17. Kastelein JJ, Ginsberg HN, Langslet G, Hovingh GK, Ceska R, Dufour R, et al. ODYSSEY FH I and FH II: 78 week results with alirocumab treatment in 735 patients with heterozygous familial hypercholesterolaemia. Eur Heart J 2015;36:2996-3003.

18. Moriarty PM, Thompson PD, Cannon CP, Guyton JR, Bergeron J, Zieve FJ, et al. Efficacy and safety of alirocumab vs. ezetimibe in statin-intolerant patients, with a statin rechallenge arm: The ODYSSEY ALTERNATIVE randomized trial. J Clin Lipidol 2015;9:758-69.

19. Koren MJ, Lundqvist P, Bolognese M, Neutel JM, Monsalvo ML, Yang J, et al. Anti-PCSK9 monotherapy for hypercholesterolemia: The MENDEL-2 randomized, controlled phase III clinical trial of evolocumab. J Am Coll Cardiol 2014;63:2531-40.

20. Blom DJ, Hala T, Bolognese M, Lillestol MJ, Toth PD, Burgess L, et al. A 52-week placebo-controlled trial of evolocumab in hyperlipidemia. N Engl J Med 2014;370:1809-19.

21. Sabatine MS, Giugliano RP, Wiviott SD, Raal FJ, Blom DJ, Robinson J, et al. Efficacy and safety of evolocumab in reducing lipids and cardiovascular events. N Engl J Med 2015;372:1500-9.

22. Raal FJ, Stein EA, Dufour R, Turner T, Civeira F, Burgess L, et al. PCSK9 inhibition with evolocumab (AMG 145) in heterozygous familial hypercholesterolaemia (RUTHERFORD-2): A randomised, double-blind, placebo-controlled trial. Lancet 2015;385:331-40.

23. Stroes E, Colquhoun D, Sullivan D, Civeira F, Rosenson RS, Watts GF, et al. Anti-PCSK9 antibody effectively lowers cholesterol in patients with statin intolerance: The GAUSS-2 randomized, placebo-controlled phase 3 clinical trial of evolocumab. J Am Coll Cardiol 2014;63:2541-8. 